\title{
LOCALIZAÇÃO DO PONTO DE ENTRADA TIBIAL
}

\section{LOCALIZATION OF THE TIBIAL ENTRY POINT}

Pedro José Labronici', Ildeu Leite Moreira Junior², Fábio Soares Lyra ${ }^{2}$, José Sergio Franco ${ }^{3}$, Rolix Hoffmann ${ }^{4}$, Paulo Roberto Barbosa de Toledo Lourenço ${ }^{5}$, Kodi Kojima ${ }^{6}$, José Soares Hungria Neto ${ }^{7}$

\section{RESUMO}

Objetivo: Verificar, através de um questionário, o ponto de entrada da haste intramedular na região proximal da tíbia. Métodos: 230 participantes que tratam fraturas da tíbia foram entrevistados. O questionário foi formulado com três segmentos que poderiam ser respondidos com um formato “sim” ou "não" e um quarto, com duas figuras que representavam uma radiografia em anteroposterior (AP) e lateral que poderiam ser respondidas com um formato “A”, "B” ou “C”. Resultados: A razão mais frequente foi a "facilidade de acesso" (67,8\%), seguida do "melhor acesso para inserção da haste” (60,9\%) e em terceiro "prevenir a dor no joelho” (27,4\%). Existiu relação significativa entre as razões de escolha do acesso como "prevenir dor no joelho" e “evitar tendinites” com os pontos A e C da figura esquemática de radiografia em AP, principalmente o ponto $C$ (crista tibial medial). Observou-se que não existiu diferença significativa nos tipos de acesso em relação ao ligamento patelar, nas figuras esquemáticas de radiografia em AP e perfil entre as faixas etárias. Conclusão: Observou-se que quanto maior a faixa etária maior a proporção de escolher a pergunta "evitar deformidade em valgo”. As razões de aspecto médico (prático) foram relacionadas com o tipo de acesso no ligamento transpatelar, enquanto que as razões de aspecto paciente (funcional) foram relacionadas com o acesso parapatelar medial. O acesso transpatelar foi escolhido pela maioria dos participantes (66,5\%).

Descritores - Fixação intramedular de fraturas; Fratura da tíbia; Ligamento patelar

\section{ABSTRACT}

Objective: To assess, through a questionnaire, the intramedullary nail entry point in the proximal aspect of the tibia. Methods: 230 attendees who treat tibial fractures were interviewed. The questionnaire was created with three sections that could be answered with "Yes" or "No" answers and a fourth section that had two figures representing anteroposterior (AP) and lateral view $x$-rays that could be answered with a format " $A$, " $B$ ” or " $C$ ". Results: The most frequent reason was "ease of access" (67.8\%), followed by the "better nail insertion access" (60.9\%) and the third one was "to prevent knee pain" (27.4\%). Reasons for choosing access so as "to prevent knee pain" and "prevent tendinitis" had a significant relationship with points " $A$ ” and " $C$ " of the schematic figure in the AP $x$-ray, especially " $C$ " (medial tibial crest). No significant difference was observed between types of access to the patellar ligament in the schematic figure of the AP and lateral x-ray among age groups. Conclusion: The greater the age, the larger the proportion choosing the question "avoid valgus deformity." Reasons from a medical (practical) perspective were related to the type of access in the transpatellar ligament, while reasons from a patient (functional) perspective were related to medial parapatellar access. Transpatellar access was chosen by most participants (66.5\%).

Keywords - Fracture fixation, Intramedullary; Tibial fractures; Patellar ligament

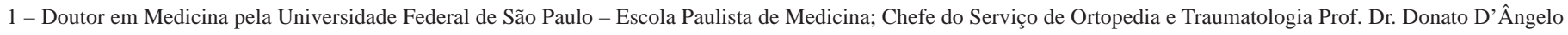
- Hospital Santa Teresa, Petrópolis/RJ, Brasil.

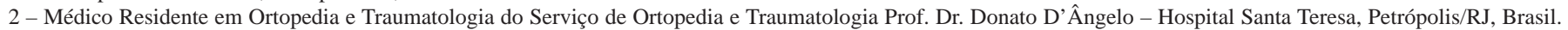

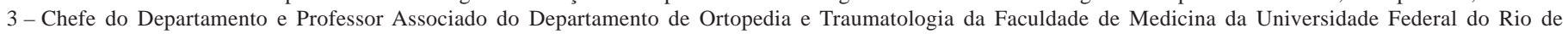
Janeiro, Brasil.

4 - Médico do Serviço de Ortopedia e Traumatologia Prof. Dr. Donato D’Ângelo - Hospital Santa Teresa, Petrópolis/RJ, Brasil.

5 - Médico Responsável pelo Grupo de Trauma do Hospital de Ipanema, Rio de Janeiro, Brasil.

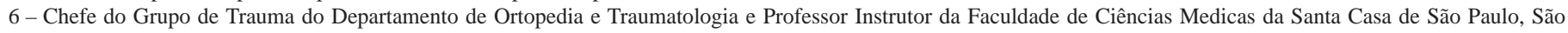
Paulo/SP, Brasil.

7 - Professor Adjunto da Faculdade de Ciências Médicas da Santa Casa de São Paulo, São Paulo/SP, Brasil.

Trabalho realizado no Serviço de Ortopedia e Traumatologia Prof. Dr. Donato D’Ângelo, Hospital Santa Teresa, Petrópolis, RJ, Brasil.

Correspondência: Av. Roberto Silveira, 187, Ap. 601 - 25685-040 - Petrópolis, RJ. E-mail: plabronici@globo.com 


\section{INTRODUÇÃO}

Fratura da diáfise da tíbia permanece como a lesão mais comum tratada pelos cirurgiões ortopédicos. Haste intramedular tem se tornado o tratamento de escolha tanto para as fraturas fechadas instáveis como para as expostas de baixa energia ${ }^{(1,2)}$. Diversos pontos de entrada na região proximal da tíbia têm sido descritos, mas o local ideal permanece como tema de discussão ${ }^{(1,3-6)}$. Vários acessos cirúrgicos podem ser utilizados para se obter o ponto de entrada incluindo o acesso parapatelar medial, lateral e a incisão transpatelar ${ }^{(1,4,7)}$. Segundo a literatura, autores têm relatado complicações como dor na região anterior do joelho, instabilidade ligamentar, necrose da gordura retropatelar, lesões de cartilagem e menisco, distrofia simpático-reflexa, alteração da marcha e tendossinovites como causas possíveis do procedimento cirúrgico ${ }^{(7-14)}$. Porém, às vezes, a dor não é aliviada com a remoção da haste, e a incisão transpatelar tem sido acusada parcialmente como a causa destas complicações ${ }^{(7-10,12)}$. O objetivo deste estudo foi verificar, através de um questionário, o ponto de entrada da haste intramedular na região proximal da tíbia, respondido no Congresso Brasileiro de Ortopedia e Traumatologia.

\section{MÉTODOS}

Durante o $40^{\circ}$ Congresso Brasileiro de Ortopedia e Traumatologia, ocorrido no Rio Grande do Sul em 2008, 230 participantes que tratam fraturas da tíbia foram entrevistados para fornecer informações. O questionário foi formulado com três segmentos que poderiam ser respondidos com um formato "sim” ou "não", e um quarto, com duas figuras que representavam uma radiografia em anteroposterior (AP) e lateral que poderia ser respondido com um formato “ $A$ ”, “ $B$ ” ou "C". Os participantes foram divididos em três categorias, segundo a faixa etária (25-35, 36-45 e > 45 anos), para verificar se a experiência do cirurgião influenciaria nos resultados.

Aos ortopedistas foi perguntado se tratavam fraturas da diáfise da tíbia com haste intramedular. Em caso positivo, foi perguntado se utilizavam um ou mais acessos. Em relação ao ligamento patelar, se utilizavam o acesso medial, lateral ou transpatelar. Também foi perguntada a razão para a seleção do acesso no ligamento patelar, ver questionário anexo.

A participação foi voluntária e as respostas foram confidenciais. Os resultados foram computados e submetidos à análise estatística.

\section{Perfil geral da casuística}

Este objetivo teve como finalidade descrever o perfil de 230 participantes, segundo o questionário de avaliação do ponto de entrada da haste intramedular na tíbia. A Tabela 1 fornece a frequência (n) e o percentual (\%) das respostas do questionário para o total da amostra.

Tabela 1 - Descrição do questionário para 230 participantes respondentes

\begin{tabular}{|c|c|c|c|}
\hline Variável & categoria & $\mathrm{n}$ & $\%$ \\
\hline \multirow{3}{*}{ Grupo etárioa } & 25 a 35 anos & 95 & 42,0 \\
\hline & 36 a 45 anos & 71 & 31,5 \\
\hline & $>45$ anos & 60 & 26,5 \\
\hline \multirow{2}{*}{$\begin{array}{l}\text { Acesso para } \\
\text { colocação da haste }\end{array}$} & Acesso único & 201 & 87,4 \\
\hline & Vários tipos & 29 & 12,6 \\
\hline \multirow{3}{*}{ Ligamento patelar } & Medial & 64 & 27,8 \\
\hline & Transpatelar & 153 & 66,5 \\
\hline & Lateral & 13 & 5,7 \\
\hline \multirow{9}{*}{$\begin{array}{l}\text { Razão para a } \\
\text { seleção do acesso } \\
\text { no ligamento }\end{array}$} & 1. Prevenir dor no joelho & 63 & 27,4 \\
\hline & $\begin{array}{l}\text { 2. Preservar a biomecânica da } \\
\text { patelofemoral }\end{array}$ & 49 & 21,3 \\
\hline & $\begin{array}{l}\text { 3. Melhor acesso para inserção } \\
\text { da haste }\end{array}$ & 140 & 60,9 \\
\hline & 4. Facilidade de acesso & 156 & 67,8 \\
\hline & 5. Localização da fratura & 21 & 9,1 \\
\hline & 6. Evitar formação de neuroma & 8 & 3,5 \\
\hline & 7. Evitar deformidade em valgo & 22 & 9,6 \\
\hline & 8. Evitar deformidade em varo & 14 & 6,1 \\
\hline & 9. Evitar tendinites & 48 & 20,9 \\
\hline \multirow{3}{*}{$\begin{array}{l}\text { Referência } \\
\text { radiográfica - } 1^{\text {b }}\end{array}$} & A & 32 & 14,4 \\
\hline & B & 149 & 66,8 \\
\hline & C & 42 & 18,8 \\
\hline \multirow{3}{*}{$\begin{array}{l}\text { Referência } \\
\text { radiográfica - } 2^{b}\end{array}$} & A & 61 & 27,4 \\
\hline & $B$ & 120 & 53,8 \\
\hline & C & 42 & 18,8 \\
\hline \multirow{2}{*}{ Comentários } & Sim & 13 & 5,7 \\
\hline & Não & 217 & 94,3 \\
\hline
\end{tabular}

\section{ANÁLISE ESTATÍSTICA}

Os testes estatísticos aplicados foram o do Quiquadrado $\left(\chi^{2}\right)$ ou o exato de Fisher, para comparação de proporções entre dados de natureza categórica. Os resultados foram apresentados em tabelas e expressos por meio de frequência (n) e percentual (\%). O critério de determinação de significância adotado foi o nível de 5\%. A análise estatística foi processada pelo software estatístico SAS ${ }^{\circledR}$ System, versão 6.04 . 


\section{RESULTADOS}

Dentre as razões para a escolha do acesso cirúrgico, observou-se que a razão mais frequente foi a "facilidade de acesso" com 67,8\%, seguida do "melhor acesso para inserção da haste" com 60,9\% e em terceiro foi "prevenir a dor no joelho” com 27,4\%. A Figura 1 ilustra a distribuição crescente das razões para a seleção do acesso no ligamento patelar.

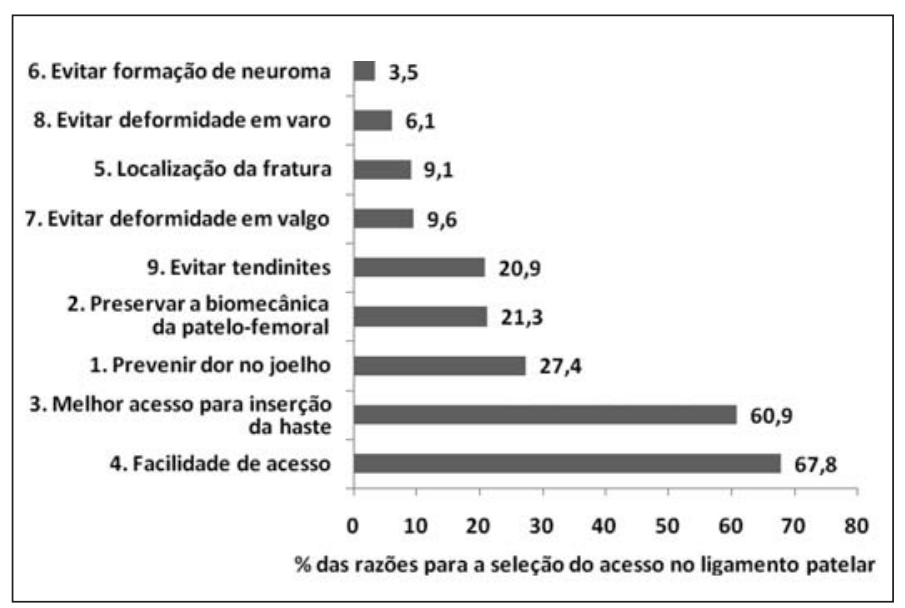

Fonte: Serviço de Ortopedia e Traumatologia do Hospital Santa Teresa. Petrópolis/RJ.

Figura 1 - Percentual das razões para a escolha do acesso no ligamento patelar

Segundo as respostas dos 230 participantes, foi verificado se existiu diferença significativa nas proporções das razões para a escolha do acesso entre as faixas etárias, quais os tipos de acessos em relação ao ligamento patelar e locais de referência esquemáticas de radiografias.

As Tabelas 2, 3, 4 e 5 fornecem a frequência (n) e o percentual (\%) das razões para a escolha do acesso segundo a faixa etária (25-35, 36-45 e > 45 anos), tipos de acessos em relação ao ligamento patelar (medial, transpatelar e lateral) e locais de referência radiográfica, conforme Figuras A, B e C do questionário anexo, respectivamente, e o correspondente nível descritivo do teste estatístico $(p)$. A análise estatística foi realizada pelo teste do $\chi^{2}$ ou pelo exato de Fisher.

Não foi possível analisar as razões "evitar formação de neuroma” e “evitar deformidades em varo” devido à baixa frequência observada.

Observou-se que existiu diferença significativa apenas na razão “evitar deformidade em valgo” ( $p=0,007)$ entre as faixas etárias, ou seja, quanto maior a faixa etária maior a proporção de escolher esta razão. Não existe diferença significativa na proporção das demais razões entre as faixas etárias, ao nível de 5\%.
Tabela 2 - Análise estatística das razões para a escolha do acesso segundo a faixa etária

\begin{tabular}{|c|c|c|c|c|c|c|c|}
\hline \multirow{2}{*}{$\frac{\text { Faixa etária }}{\text { Razão para a seleção do acesso }}$} & \multicolumn{2}{|c|}{25 a 35 anos } & \multicolumn{2}{|c|}{36 a 45 anos } & \multicolumn{2}{|c|}{$>45$ anos } & \multirow{2}{*}{$p$} \\
\hline & $\mathrm{n}$ & $\%$ & $\mathrm{n}$ & $\%$ & $\mathrm{n}$ & $\%$ & \\
\hline 1. Prevenir dor no joelho & 28 & 29,5 & 15 & 21,1 & 20 & 33,3 & 0,27 \\
\hline $\begin{array}{l}\text { 2. Preservar a biomecânica da } \\
\text { patelofemoral }\end{array}$ & 18 & 19,0 & 12 & 16,9 & 18 & 30,0 & 0,14 \\
\hline $\begin{array}{l}\text { 3. Melhor acesso para inserção } \\
\text { da haste }\end{array}$ & 60 & 63,2 & 46 & 64,8 & 32 & 53,3 & 0,35 \\
\hline 4. Facilidade de acesso & 66 & 69,5 & 51 & 71,8 & 37 & 61,7 & 0,43 \\
\hline 5. Localização da fratura & 8 & 8,4 & 5 & 7,0 & 8 & 13,3 & 0,43 \\
\hline 6. Evitar formação de neuroma & 2 & 2,1 & 1 & 1,4 & 4 & 6,7 & pc \\
\hline 7. Evitar deformidade em valgo & 3 & 3,2 & 8 & 11,3 & 11 & 18,3 & 0,007 \\
\hline 8. Evitar deformidade em varo & 4 & 4,2 & 4 & 5,6 & 6 & 10,0 & pc \\
\hline 9. Evitar tendinites & 19 & 20,0 & 13 & 18,3 & 16 & 26,7 & 0,47 \\
\hline
\end{tabular}

pc: Poucos casos.

Fonte: Serviço de Ortopedia e Traumatologia do Hospital Santa Teresa. Petrópolis/RJ.

Tabela 3 - Análise estatística das razões para seleção do acesso em relação ao ligamento patelar

\begin{tabular}{|c|c|c|c|c|c|c|c|}
\hline \multirow{2}{*}{$\frac{\text { Ligamento patelar }}{\text { Razão para a seleção do acesso }}$} & \multicolumn{2}{|c|}{ Medial } & \multicolumn{2}{|c|}{ Transpatelar } & \multicolumn{2}{|c|}{ Lateral } & \multirow{2}{*}{$P^{*}$} \\
\hline & $\mathrm{n}$ & $\%$ & $\mathrm{n}$ & $\%$ & $\mathrm{n}$ & $\%$ & \\
\hline 1. Prevenir dor no joelho & 38 & 59,4 & 16 & 10,5 & 9 & 69,2 & $<0,0001$ \\
\hline $\begin{array}{l}\text { 2. Preservar a biomecânica da } \\
\text { patelofemoral }\end{array}$ & 27 & 42,2 & 14 & 9,2 & 8 & 61,5 & $<0,0001$ \\
\hline $\begin{array}{l}\text { 3. Melhor acesso para inserção } \\
\text { da haste }\end{array}$ & 23 & 35,9 & 112 & 73,2 & 5 & 38,5 & $<0,0001$ \\
\hline 4. Facilidade de acesso & 29 & 45,3 & 122 & 79,7 & 5 & 38,5 & $<0,0001$ \\
\hline 5. Localização da fratura & 4 & 6,3 & 16 & 10,5 & 1 & 7,7 & 0,32 \\
\hline 6. Evitar formação de neuroma & 2 & 3,1 & 5 & 3,3 & 1 & 7,7 & $\mathrm{pc}$ \\
\hline 7. Evitar deformidade em valgo & 5 & 7,8 & 16 & 10,5 & 1 & 7,7 & 0,54 \\
\hline 8. Evitar deformidade em varo & 3 & 4,7 & 10 & 6,5 & 1 & 7,7 & $\mathrm{pc}$ \\
\hline 9. Evitar tendinites & 33 & 51,6 & 5 & 3,3 & 10 & 76,9 & $<0,0001$ \\
\hline
\end{tabular}

Para fins de análise, não foi considerado o acesso ao ligamento lateral devido à baixa frequência observada $(\mathrm{n}=13)$. Observou-se que as razões de aspecto médico (prático) como "melhor acesso para inserção da haste" e "facilidade de acesso" foi relacionada significativamente com o acesso transpatelar, enquanto que as razões de aspecto paciente (funcional) como "prevenir dor no joelho”, "preservar a biomecânica” e "evitar tendinites” foram relacionadas significativamente com o acesso parapatelar medial. 
Tabela 4 - Análise estatística das razões para seleção do acesso segundo o local de referência radiográfica - figura esquemática de radiografia em AP

\begin{tabular}{|c|c|c|c|c|c|c|c|}
\hline \multirow{2}{*}{$\frac{\text { Referência radiográfica } 1}{\text { Razão para a seleção do acesso }}$} & \multicolumn{2}{|c|}{ Ponto $\mathrm{A}$} & \multicolumn{2}{|c|}{ Ponto B } & \multicolumn{2}{|c|}{ Ponto C } & \multirow{2}{*}{$p$} \\
\hline & $\mathrm{n}$ & $\%$ & $\mathrm{~N}$ & $\%$ & $\mathrm{n}$ & $\%$ & \\
\hline 1. Prevenir dor no joelho & 10 & 31,3 & 31 & 20,8 & 20 & 47,6 & 0,002 \\
\hline $\begin{array}{l}\text { 2. Preservar a biomecânica da } \\
\text { patelofemoral }\end{array}$ & 9 & 28,1 & 27 & 18,1 & 12 & 28,6 & 0,21 \\
\hline $\begin{array}{l}\text { 3. Melhor acesso para inserção } \\
\text { da haste }\end{array}$ & 16 & 50,0 & 96 & 64,4 & 23 & 54,8 & 0,22 \\
\hline 4. Facilidade de acesso & 19 & 59,4 & 108 & 72,5 & 25 & 59,5 & 0,14 \\
\hline 5. Localização da fratura & 2 & 6,3 & 15 & 10,1 & 4 & 9,5 & 0,88 \\
\hline 6. Evitar formação de neuroma & 2 & 6,3 & 4 & 2,7 & 2 & 4,8 & $\mathrm{pc}$ \\
\hline 7. Evitar deformidade em valgo & 2 & 6,3 & 14 & 9,4 & 6 & 14,3 & 0,55 \\
\hline 8. Evitar deformidade em varo & 2 & 6,3 & 10 & 6,7 & 2 & 4,8 & $\mathrm{pc}$ \\
\hline 9. Evitar tendinites & 11 & 34,4 & 19 & 12,8 & 16 & 38,1 & $<0,0001$ \\
\hline
\end{tabular}

\section{pc: Poucos casos.}

Fonte: Serviço de Ortopedia e Traumatologia do Hospital Santa Teresa. Petrópolis/RJ.

Tabela 5 - Análise estatística das razões para seleção do acesso segundo o local de referência radiográfica - figura esquemática de radiografia em perfil

\begin{tabular}{l|c|c|c|c|c|c|c}
\hline \multicolumn{1}{c|}{ Referência radiográfica 2 } & \multicolumn{2}{|c|}{ Ponto A } & \multicolumn{2}{|c|}{ Ponto B } & \multicolumn{2}{c|}{ Ponto C } & \multirow{2}{*}{$\boldsymbol{p}$} \\
\cline { 1 - 4 } Razão para a seleção do acesso & $\mathbf{n}$ & $\%$ & $\mathbf{n}$ & $\%$ & $\mathbf{n}$ & $\%$ & \\
\hline 1. Prevenir dor no joelho & 15 & 24,6 & 33 & 27,5 & 13 & 31,0 & 0,77 \\
\hline $\begin{array}{l}\text { 2. Preservar a biomecânica da } \\
\text { patelofemoral }\end{array}$ & 11 & 18,0 & 26 & 21,7 & 11 & 26,2 & 0,61 \\
\hline $\begin{array}{l}\text { 3. Melhor acesso para inserção } \\
\text { da haste }\end{array}$ & 34 & 55,7 & 73 & 60,8 & 28 & 66,7 & 0,53 \\
\hline 4. Facilidade de acesso & 40 & 65,6 & 84 & 70,0 & 28 & 66,7 & 0,81 \\
\hline 5. Localização da fratura & 5 & 8,2 & 12 & 10,0 & 4 & 9,5 & 0,92 \\
\hline 6. Evitar formação de neuroma & 1 & 1,6 & 4 & 3,3 & 3 & 7,1 & $\mathrm{pc}$ \\
\hline 7. Evitar deformidade em valgo & 2 & 3,3 & 9 & 7,5 & 11 & 26,2 & $<0,0001$ \\
\hline 8. Evitar deformidade em varo & 1 & 1,6 & 5 & 4,2 & 8 & 19,1 & $\mathrm{pc}$ \\
\hline 9. Evitar tendinites & 14 & 23,0 & 23 & 19,2 & 9 & 21,4 & 0,82 \\
\hline
\end{tabular}

\section{pc: Poucos casos.}

Fonte: Serviço de Ortopedia e Traumatologia do Hospital Santa Teresa. Petrópolis/RJ.

Verificou-se que existiu relação significativa entre as razões de escolha do acesso como "prevenir dor no joelho" $(p=0,002)$ e "evitar tendinites" ( $<<0,0001)$ com os pontos A e $C$ da figura esquemática de radiografia em AP, principalmente o ponto C (crista tibial medial). Não foi encontrada diferença significativa na proporção das demais razões entre os locais da figura esquemática de radiografia em AP, ao nível de 5\%. Embora não tenha significância, existiu predominância das razões para o acesso de aspecto médico como "melhor acesso para inserção" e "facilidade de acesso" no local de referência radiográfica - ponto B.
Percebeu-se que existiu relação significativa apenas entre a razão "evitar deformidade em valgo" ( $p<$ $0,0001)$ com o ponto $C$ o local de referência radiográfica - figura esquemática de radiografia em perfil. Não existiu diferença significativa na proporção das demais razões entre os locais de referência radiográfica, ao nível de $5 \%$.

A Tabela 6 fornece a frequência (n) e o percentual (\%) do tipo de acesso em relação ao ligamento patelar (medial, transpatelar e lateral) e local de referência radiográfica (Figuras A, B e C) do questionário, segundo a faixa etária (25-35, 36-45 e > 45 anos), e o correspondente nível descritivo do teste estatístico $(p)$. A análise estatística foi realizada pelo teste do $\chi^{2}$.

Tabela 6 - Análise estatística do tipo de acesso em relação ao ligamento patelar e referência radiográfica segundo a faixa etária

\begin{tabular}{|c|c|c|c|c|c|c|c|c|}
\hline \multirow{2}{*}{ Variável } & \multirow{2}{*}{ Categoria } & \multicolumn{2}{|c|}{25 a 35 anos } & \multicolumn{2}{|c|}{36 a 45 anos } & \multicolumn{2}{|c|}{$>45$ anos } & \multirow{2}{*}{$p$} \\
\hline & & $\mathrm{n}$ & $\%$ & $\mathrm{n}$ & $\%$ & $\mathrm{n}$ & $\%$ & \\
\hline \multirow{3}{*}{$\begin{array}{l}\text { Ligamento } \\
\text { patelar }\end{array}$} & Medial & 26 & 27,4 & 20 & 28,2 & 17 & 28,3 & \multirow{3}{*}{0,78} \\
\hline & Transpatelar & 62 & 65,3 & 49 & 69,0 & 39 & 65,0 & \\
\hline & Lateral & 7 & 7,4 & 2 & 2,8 & 4 & 6,7 & \\
\hline \multirow{3}{*}{$\begin{array}{c}\text { Referência } \\
\text { radiográfica } \\
\text { Figura } 1\end{array}$} & Ponto A & 15 & 15,8 & 6 & 9,0 & 10 & 17,5 & \multirow{3}{*}{0,33} \\
\hline & Ponto B & 65 & 68,4 & 49 & 73,1 & 33 & 57,9 & \\
\hline & Ponto C & 15 & 15,8 & 12 & 17,9 & 14 & 24,6 & \\
\hline \multirow{3}{*}{$\begin{array}{c}\text { Referência } \\
\text { radiográfica } \\
\text { Figura } 2\end{array}$} & Ponto A & 26 & 27,4 & 19 & 28,4 & 15 & 26,3 & \multirow{3}{*}{0,65} \\
\hline & Ponto B & 55 & 57,9 & 35 & 52,2 & 28 & 49,1 & \\
\hline & Ponto C & 14 & 14,7 & 13 & 19,4 & 14 & 24,6 & \\
\hline
\end{tabular}

Fonte: Serviço de Ortopedia e Traumatologia do Hospital Santa Teresa. Petrópolis/RJ.

Observou-se que existiu diferença significativa entre o local de referência da figura esquemática de radiografia em AP ( $<<0,0001)$ com o tipo de acesso em relação ao ligamento patelar, ou seja, o acesso lateral predominou o ponto A, o transpatelar o ponto B e o medial o ponto $\mathrm{C}$ e $\mathrm{B}$, nesta ordem. Notou-se que não existiu diferença significativa nos tipos de acesso em relação ao ligamento patelar ( $p=0,78)$, nas figuras esquemáticas de radiografia em AP $(p=0,33)$ e perfil $(p=0,65)$ entre as faixas etárias, ao nível de $5 \%$.

\section{DISCUSSÃO}

A haste intramedular é o tratamento de escolha para as fraturas da diáfise da tíbia fechadas instáveis e as expostas de baixa energia ${ }^{(2,15)}$. O sucesso da colocação da haste tibial depende do local e do ângulo de inserção $0^{(16)}$. 
O ponto de entrada ideal da haste tibial tem sido motivo de muitas discussões. Freeman e Johnson ${ }^{(16)}$ demonstraram que o ângulo do eixo longitudinal da haste e o fragmento proximal é mais importante que o ponto de inserção para a manutenção do alinhamento ósseo após o tratamento com haste na fratura proximal da tíbia. Entretanto, um ponto de entrada impróprio, pode levar a desvio da haste contra o córtex proximal. Alms ${ }^{(3)}$ recomendou um ponto de entrada lateral sobre a superfície extrassinovial anterossuperior. Chapman ${ }^{(17)}$ determinou o ponto de entrada acima da tuberosidade anterior da tíbia através da via transpatelar. Tornetta et al $^{(6)}$ descreveram uma zona de segurança anatômica para a colocação da haste em uma área de aproximadamente $22,9 \mathrm{~mm}$. Este estudo indicou que a lesão do menisco lateral ou medial poderia ser o responsável pela dor no joelho. McConnell et al ${ }^{(5)}$ demonstraram que o ponto de entrada ideal é medial à espinha tibial lateral na visão anteroposterior e imediatamente adjacente à margem anterior da superfície articular na visão lateral.

A experiência do cirurgião foi analisada, segundo as razões da seleção do acesso, em relação à faixa etária (25-35, 36-45 e > 45 anos). Em todas as faixas etárias, a maior preocupação foi com o "melhor acesso para a inserção da haste" e a "facilidade de acesso". Porém, observou-se que existiu preocupação maior em relação à deformidade em valgo, ou seja, quanto maior a faixa etária maior a proporção de escolher esta razão. Lang et $a^{(18)}$ observaram que a consolidação viciosa em valgo nas fraturas da tíbia foi devida a um ponto de entrada medial e um ângulo de inserção da haste direcionada lateralmente no fragmento proximal.

Para se acessar o ponto de entrada na tíbia, a incisão pode ser a parapatelar lateral ou medial e a transpatelar. Vários autores têm relatado alta incidência de dor no joelho com o acesso transpatelar após inserção da haste intramedular e sugerem que o acesso paratendinoso ajuda a diminuir este sintoma ${ }^{(7,8,10,11,13,19,20)}$. Nossos resultados demonstraram que, independente da faixa etária, a maioria dos participantes utiliza o acesso transpatelar, e que somente $27,4 \%$ expressaram preocupação com a dor no joelho. Entretanto, dos participantes que escolheram o acesso parapatelar medial, 59,4\% expressaram a preocupação em prevenir a dor no joelho. No contexto geral, observou-se que as razões para a escolha do acesso foram divididas em dois aspectos: o primeiro, que chamamos de médico (prático), elegeram como "melhor acesso para a inserção da haste" (60,9\%) e "facilidade de acesso" (67,8\%) e foram relacionadas com o tipo acesso transpatelar. Quanto às razões de aspecto paciente (funcional), isto é, preocupados com o resultado final, escolheram as razões como "prevenir a dor no joelho" (27,4\%), "preservar a biomecânica da patelofemoral” (21,3\%) e “evitar tendinites”(20,9\%) foram relacionados com o tipo de acesso parapatelar medial. Althausen et $a^{(21)}$ reservaram o acesso transpatelar para as tíbias com o ponto de entrada ideal localizado na posição central e atrás do tendão patelar nas fraturas proximais da tíbia, deixando o acesso parapatelar para os outros padrões das fraturas. Também preconizam a utilização de hastes com pouca curvatura (curva de Herzog) para conseguir o melhor alinhamento possível.

McConnell et $a l^{(5)}$ demonstraram que o ponto de entrada ideal é medial à espinha tibial lateral na visão anteroposterior e imediatamente adjacente à margem anterior da superfície articular na visão lateral. Samuelson et $a^{(22)}$ encontraram bons resultados utilizando o acesso parapatelar medial e o transpatelar quando evitavam o local de inserção lateral ao tubérculo tibial. Althausen et $a l^{(21)}$ concluíram que existem variações anatômicas entre o tendão patelar e a espinha tibial lateral. Esta variação implica que uma rotina do acesso cirúrgico pode não dar a direção ideal para o ponto de entrada. Avaliação pré-operatória através da fluoroscopia pode direcionar o cirurgião para o melhor acesso para alcançar o ponto de entrada. Nossos resultados demonstraram que a maioria dos participantes escolheu o ponto B da figura esquemática de radiografia tanto em AP como em perfil (66,8\% e $53,8 \%$, respectivamente) e somente 13 participantes $(5,7 \%)$ teceram alguns comentários em relação ao local de inserção da haste. Observou-se que existiu relação entre as razões de aspecto do paciente (funcional) como "prevenir a dor no joelho" e "evitar tendinites" com os pontos A e C e predominância em relação às razões de aspecto médico (prático) como "melhor acesso para inserção" e " "facilidade de acesso" com o ponto B da figura esquemática de radiografia em AP. Quanto às razões para a seleção do acesso segundo a figura esquemática de radiografia em perfil, existiu relação apenas entre a razão "evitar deformidade em valgo" com o ponto C.

\section{CONCLUSÃO}

Verificou-se que quanto maior a faixa etária maior a proporção de escolher a pergunta "evitar deformidade em valgo”. As razões de aspecto médico (prático) como "melhor acesso para inserção da haste" e "facilidade de acesso" foram relacionadas com o acesso transpatelar, enquanto que as razões de aspecto paciente (funcional) como "prevenir dor no joelho", "preservar a biomecânica" e "evitar tendinites" foram relacionadas com o acesso parapatelar medial. O acesso transpatelar foi escolhido pela maioria dos participantes (66,5\%). 


\section{ANEXO}

Questionário de avaliação do ponto de entrada da haste intramedular na tíbia

Nome:

Instituição:

Idade:

Acesso(s) para a colocação da haste intramedular:

$\square$ Utiliza acesso único

$\square$ Utiliza vários tipos de acessos

Em relação ao ligamento patelar:

$\square$ Medial

$\square$ Transpatelar

$\square$ Lateral

Razão para a seleção do acesso no ligamento patelar:

$\square$ Prevenir dor no joelho

$\square$ Preservar a biomecânica da patelofemoral

$\square$ Melhor acesso para inserção da haste

$\square$ Facilidade de acesso

$\square$ Localização da fratura

$\square$ Evitar formação de neuroma

$\square$ Evitar deformidade em valgo

$\square$ Evitar deformidade em varo

$\square$ Evitar tendinites

$\square$ Outras
Referência radiográfica para o ponto de entrada da haste intramedular

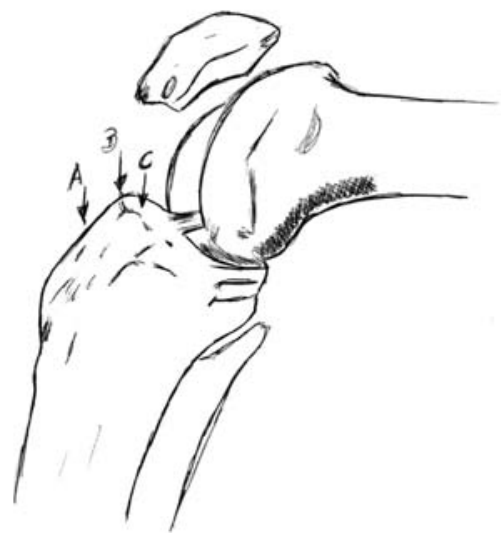

$\mathrm{B} \square$

$\mathrm{C} \square$

Comentários:

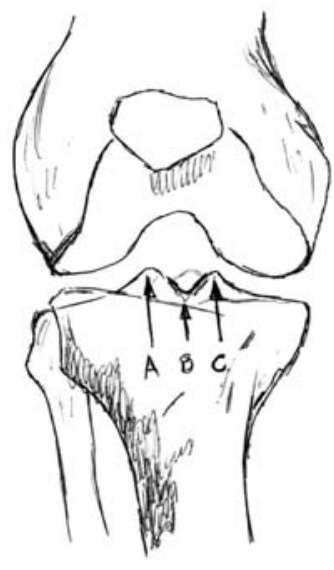
$\mathrm{C} \square$
$\mathrm{A} \square$
$\mathrm{B} \square$

Comentários: 


\section{REFERÊNCIAS}

1. Court-Brown CM, Christie J, McQueen MM. Closed intramedullary tibial nailing. Its use in closed and type I open fractures. J Bone Joint Surg Br. 1990;72(4):605-11.

2. Tornetta $P$ 3rd. Technical considerations in the surgical management of tibial fractures. Instr Course Lect. 1997;46:271-80.

3. Alms M. Medullary nailing for fracture of the shaft of the tibia. J Bone Joint Surg Br. 1962;44:328-39.

4. Buehler KC, Green J, Woll TS, Duwelius PJ. A technique for intramedullary nailing of proximal third tibia fractures. J Orthop Trauma. 1997;11(3):218-23.

5. McConnell T, Tornetta P 3rd, Tilzey J, Casey D. Tibial portal placement: the radiographic correlate of the anatomic safe zone. J Orthop Trauma. 2001;15(3):207-9.

6. Tornetta P 3rd, Riina J, Geller J, Purban W. Intraarticular anatomic risks of tibial nailing. J Orthop Trauma. 1999;13(4):247-51.

7. Yu SW, Tu YK, Fan KF, Su JY. Anterior knee pain after intramedullary tibial nailing. Changgeng Yi Xue Za Zhi. 1999;22(4):604-8.

8. Court-Brown CM, Gustilo T, Shaw AD. Knee pain after intramedullary tibial nailing: its incidence, etiology, and outcome. J Orthop Trauma. 1997;11(2):103-5.

9. Hardy JR, Gregg PJ, Wood C. Knee pain after tibial nailing. J Bone Joint Surg Br. 1996;78(3):510-1.

10. Keating JF, Orfaly R, O'Brien PJ. Knee pain after tibial nailing. J Orthop Trauma. 1997;11(1):10-3.

11. Koval KJ, Clapper MF, Brumback RJ, Ellison PS Jr, Poka A, Bathon GH, et al. Complications of reamed intramedullary nailing of the tibia. J Orthop Trauma.1991;5(2):184-9.
12. McCormack D, Mulcahy D, McElwain J. Knee pain after tibial nailing. J Bone Joint Surg Br. 1996;78(3):511.

13. Orfaly R, Keating JE, O'Brien PJ. Knee pain after tibial nailing: does the entry point matter? J Bone Joint Surg Br. 1995;77(6):976-7.

14. Hernigou P, Cohen D. Proximal entry for intramedullary nailing of the tibia. The risk of unrecognised articular damage. J Bone Joint Surg Br. 2000;82(1):33-41.

15. Gregory P, Sanders R. The treatment of closed, unstable tibial shaft fractures with unreamed interlocking nails. Clin Orthop Relat Res. 1995;(315):48-55.

16. Freedman EL, Johnson EE. Radiographic analysis of tibial fracture malalignment following intramedullary nailing. Clin Orthop Relat Res. 1995;(315):25-33.

17. Chapman MW. Fractures of the tibia and fibula. In: Operative orthopaedics. 2nd ed. Philadelphia: J. B. Lippincott Company; 1993..

18. Lang GJ, Cohen BE, Bosse MJ, Kellam JF. Proximal third tibial shaft fractures. Should they be nailed? Clin Orthop Relat Res. 1995;(315):64-74.

19. Väistö O, Toivanen J, Paakkala T, Järvelä T, Kannus $P$, Järvinen M. Anterior knee pain after intramedullary nailing of a tibial shaft fracture: an ultrasound study of the patellar tendons of 36 patients. J Orthop Trauma. 2005;19(5):311-6.

20. Toivanen JA, Väistö O, Kannus P, Latvala K, Honkonen SE, Järvinen MJ. Anterior knee pain after intramedullary nailing of fractures of the tibial shaft. $A$ prospective, randomized study comparing two different nail-insertion techniques. J Bone Joint Surg Am. 2002;84(4):580-5.

21. Althausen PL, Neiman R, Finkemeier CG, Olson SA. Incision placement for intramedullary tibial nailing: an anatomic study. J Orthop Trauma. 2002;16(10):687-90.

22. Samuelson MA, McPherson EJ, Norris L. Anatomic assessment of the proper insertion site for a tibial intramedullary nail. J Orthop Trauma. 2002;16(1):23-5. 